\title{
Haploinsufficiency of immune checkpoint receptor CTLA4 induces a distinct neuroinflammatory disorder
}

\author{
Matthew K. Schindler, ${ }^{1,2}$ Stefania Pittaluga, ${ }^{3}$ Yoshimi Enose-Akahata, ${ }^{4}$ Helen C. Su, ${ }^{5}$ V. Koneti Rao, ${ }^{6}$ Amy Rump, ${ }^{6}$ \\ Steven Jacobson, ${ }^{4}$ Irene Cortese, ${ }^{1}$ Daniel S. Reich, ${ }^{1,2}$ and Gulbu Uzel ${ }^{6}$ \\ 1'Neuroimmunology Clinic and ${ }^{2}$ Translational Neuroradiology Section, National Institutes of Neurological Diseases and Stroke (NINDS), ${ }^{3}$ Laboratory of Pathology, National Cancer Institute (NCI), \\ ${ }^{4}$ Viral Immunology Section, NINDS, and ${ }^{5}$ Human Immunological Diseases Section and ${ }^{6}$ Immunopathogenesis Section, Laboratory of Clinical Immunology and Microbiology, National Institute of Allergy and \\ Infectious Disease (NIAID), NIH, Bethesda, Maryland, USA.
}

\begin{abstract}
BACKCROUND. Cytotoxic T lymphocyte antigen 4 (CTLA4) is essential for immune homeostasis. Genetic mutations causing haploinsufficiency (CTLA4h) lead to a phenotypically heterogenous, immune-mediated disease that can include neuroinflammation. The neurological manifestations of CTLA4h are poorly characterized.
\end{abstract}

METHODS. We performed an observational natural history study of 50 patients with CTLA4h who were followed at the NIH. We analyzed clinical, radiological, immunological, and histopathological data.

RESULTS. Evidence for neuroinflammation was observed in $32 \%$ ( $n=16$ of 50 ) of patients in this cohort by magnetic resonance imaging (MRI) and/or by cerebrospinal fluid analysis. Clinical symptoms were commonly absent or mild in severity, with headaches as the leading complaint $(n=13$ of 16). The most striking findings were relapsing, large, contrastenhancing focal lesions in the brain and spinal cord observed on MRI. We detected inflammation in the cerebrospinal fluid and leptomeninges before the parenchyma. Brain biopsies of inflammatory lesions from 10 patients showed perivascular and intraparenchymal mixed cellular infiltrates with little accompanying demyelination or neuronal injury.

CONCLUSIONS. Neuroinflammation due to CTLA4h is mediated primarily by an infiltrative process with a distinct and striking dissociation between clinical symptoms and radiological findings in the majority of patients.

FUNDING. NIAID, NIH, Division of Intramural Research, NINDS, NIH, Division of Intramural Research, and the National Multiple Sclerosis Society-American Brain Foundation.

TRIAL REGISTRATION. ClinicalTrials.gov NCT00001355.

\section{Introduction}

Cytotoxic T lymphocyte antigen 4 (CTLA4) is an essential protein in immune homeostasis that is constitutively expressed on regulatory $\mathrm{T}$ cells (Tregs) and upregulated on effector T cells upon activation $(1,2)$. CTLA4 outcompetes CD28 for CD80/CD86, leading to downregulation of immune activation. This physiological brake on the immune response led to coinage of the term "checkpoint" to describe the CTLA4 signaling pathway.

The CTLA4 signaling pathway has been exploited in the treatment of autoimmune arthritis with the soluble CTLA4 fusion protein abatacept (3). In contrast, monoclonal antibody blockade of CTLA4 with ipilimumab enhances the antitumor activity of $\mathrm{T}$ cells. Nevertheless, while revolutionizing the treatment of advanced melanoma (4), adverse events in patients with cancer treated with ipilimumab alone, or in combination with other checkpoint inhibitors, have been described (5). Peripheral nervous system-related (PNS-related)

Conflict of interest: The authors have declared that no conflict of interest exists. Copyright: ( 2020, American Society for Clinical Investigation.

Submitted: January 17, 2020; Accepted: July 14, 2020; Published: September 21, 2020.

Reference information: J Clin Invest. 2020;130(10):5551-5561.

https://doi.org/10.1172/JCl135947. adverse events following iatrogenic CTLA4 blockade include acute and chronic inflammatory polyneuropathies and myasthenia gravis (6-10). Case reports of aseptic meningitis, temporal arteritis, neurosarcoidosis, necrotic myelopathy, autoimmune encephalitis, and central nervous system (CNS) demyelination have also been described with the clinical use of checkpoint inhibitors $(8,9,11-14)$.

Homozygous Ctla4-deficient B57BL/6 and BALB/c mice develop extensive $\mathrm{T}$ cell-mediated infiltrates, multiorgan damage, and early death (15), whereas heterozygous mice remain healthy. Discovery of uniallelic germline mutations of CTLA4 in individuals with systemic immune dysregulation followed the mouse models by almost 2 decades $(16,17)$, with findings of diminished CTLA4 expression and Treg function similar to those in the $\mathrm{Ctla4}^{-/-}$mice. Clinical manifestations of human CTLA4 haploinsufficiency (CTLA4h) include hypogammaglobulinemia, recurrent infections, autoimmune cytopenias, autoimmune endocrinopathies, and, most critically, abnormal lymphoproliferation and lymphocytic infiltration of the gastrointestinal system, lungs, and brain. Penetrance and expressivity are variable in patient populations as well as within the same family $(16,17)$.

Here, we report the first longitudinal study to our knowledge of the neurological findings from an observational natural history 


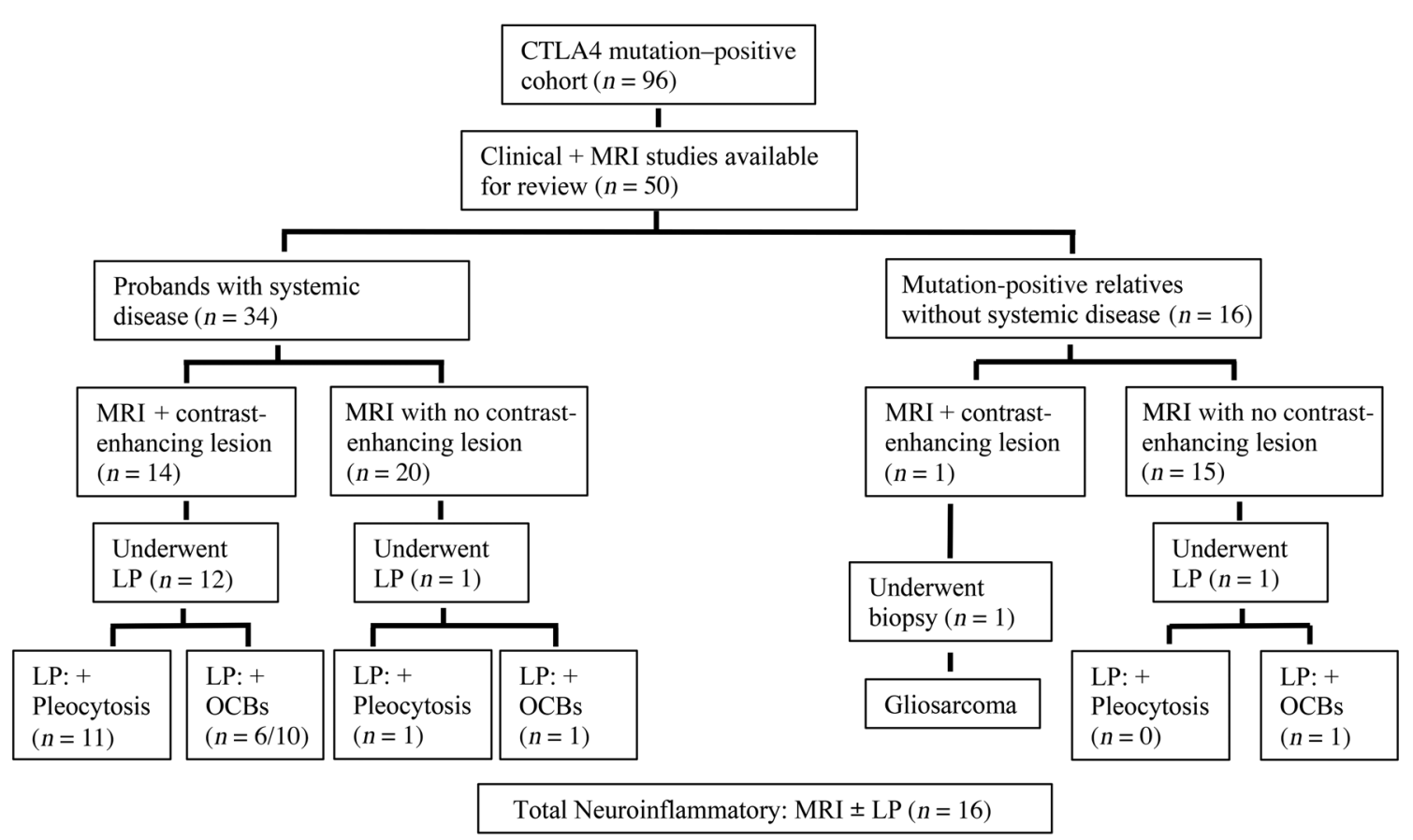

Figure 1. Flowchart summarizing participant categorization in this study. The diagram shows the inclusion and categorization of patients included for study in this analysis. Probands with systemic disease include individuals with CTLA4 mutations and any immune-mediated disease.

study of 50 patients (median follow-up: 2.3 years) with genetically defined mutations in CTLA4. All patients were evaluated at the NIH Clinical Center (NIHCC). We performed analysis of clinical, radiological, immunological, and histopathological data to determine the role of CTLA4 in immune homeostasis of the CNS.

\section{Results}

\section{Demographic and clinical data}

From a cohort of 96 patients genetically confirmed for uniallelic germline CTLA4 mutations, 50 patients $(n=24$ females and 26 males) from 23 unrelated families were selected for further analysis on the basis of the availability of imaging and clinical data (Figure 1). The average age of the cohort was 32.5 years (range: $7-81$ years). The spectrum of clinical disease ranged from asymptomatic to multiorgan system involvement with immune deficiency, immune dysregulation, and autoimmunity.

Patients were grouped on the basis of the presence of neuroinflammation defined by findings on magnetic resonance imaging (MRI) or cerebrospinal fluid (CSF) analyses, but not necessarily by clinical symptomatology. Neuroinflammation on MRI required the presence of at least 1 contrast-enhancing parenchymal lesion (1 patient with a contrast-enhancing lesion observed on MRI that was biopsied and diagnosed as gliosarcoma is not included in the neuroinflammation group). CSF inflammation required either leukocytosis or the presence of oligoclonal bands (OCBs). Sixteen patients met these criteria for neuroinflammation ( $n=14$ by MRI and/or CSF analysis and $n=2$ by CSF analysis only). The demographic and clinical information for the neuroinflammatory and non-neuroinflammatory groups are summarized in Table 1.

The median age of the patients at discovery of CNS inflammation was 18.8 years (range: 9-34 years). Only 1 patient had
CNS inflammation identified at the time of clinical disease onset, which occurred concurrently with lung inflammation. In the other patients, neuroinflammation was found at a median of 9.2 years (range: 0-25 years) after the first clinical evidence of autoimmunity or systemic organ inflammation. The most common coexisting immune-mediated signs in patients with neuroinflammation were autoimmune cytopenias and hypogammaglobulinemia, although most patients had a history of recurrent sinopulmonary infections. Individual patients' age, family, sex, and organs with evidence of autoimmunity or immune-mediated disease attributed to CTLA4h for those with neuroinflammation are shown in Supplemental Table 1 (Supplemental material available online with this article; https://doi.org/10.1172/JCI135947DS1).

\section{Neurological symptoms in CTLA4h}

Headache was the most common neurological complaint $(n=24$ of $50,48 \%$ ) in this cohort and was more common in patients with objective evidence of neuroinflammation (94\%) than in those without (26\%). The headaches were migraine-like, nonpositional, hemi- or holocephalic, and lacked any concomitant alarming features such as double vision or projectile vomiting. In patients with neuroinflammation, headaches were the most common primary complaint that triggered neuroimaging ( $n=12$ of $16,75 \%$ ), which led to the discovery of contrast-enhancing lesions on MRI. Seizure was the second most common neurological sign ( $n=12$ of 50, $24 \%$; 9 of $16,56 \%$, in patients with neuroinflammation). Seizures were typically focal in onset and occurred in patients who had large supratentorial inflammatory lesions, lesions near the cortex, or lesions in the medial temporal lobe. Seven of the 9 patients (78\%) with seizures were successfully weaned from antiepileptic treatment following resolution of the inflammatory lesions. The 3 patients without neuroinflammatory lesions ( $n=3$ of $26,12 \%$ ) 
Table 1. Summary of CTLA4h-related clinical disease findings

\begin{tabular}{lcc} 
& CNS inflammation & No CNS inflammation \\
No. of patients & $n=16$ & $n=34$ \\
Sex & $n=10 \mathrm{M} ; n=6 \mathrm{~F}$ & $n=16 \mathrm{M} ; n=14 \mathrm{~F}$ \\
Age (yr, range) at first clinical immune-mediated & $9.6(2-22)$ & $20.3(2-59)$ \\
symptom/sign & & $\mathrm{NA}$ \\
Age (yr, range) at CNS inflammation diagnosis & $18.8(9-34)$ & $\mathrm{NA}$ \\
Delay (yr, range) to CNS inflammation diagnosis & $9.2(0-25)$ & $36.9(7-81)$ \\
Mean age at NIH evaluation (yr, range) & $23.8(15-42)$ & $32 \%(n=11)$ \\
Percentage with inflammatory gastrointestinal disease & $81 \%(n=13)$ & $24 \%(n=8)$ \\
Percentage with inflammatory lung disease & $88 \%(n=14)$ & $38 \%(n=13)$ \\
Percentage with immune-mediated cytopenias & $94 \%(n=15)$ & $29 \%(n=10)$ \\
Percentage with hypogammaglobulinemia & $94 \%(n=15)$ & $24 \%(n=8)$ \\
Percentage with endocrinopathy & $44 \%(n=7)$ & $9 / 9$ \\
Neurological symptoms & No. of patients at first MRI time point/at any MRI time point \\
\hline Headache & $12 / 15$ & $3 / 3$ \\
\hline Seizure & $4 / 9$ & $0 / 0$ \\
\hline Blurry vision & $5 / 6$ & $0 / 0$ \\
\hline Focal weakness & $0 / 2$ & $0 / 0$ \\
\hline Gait imbalance & $0 / 4$ & 22 \\
\hline Screening & 2 & \\
M, males; F, females; NA, not applicable. & & \\
\hline
\end{tabular}

3 patients, who had signal abnormality that extended more than 3 vertebral bodies in length. MRI scans from 3 patients demonstrating large contrast-enhancing lesions are shown in Figure 2, A-H. Importantly, the clinical signs and symptoms at the time of the MRI scans shown in Figure 2, A-H, were nonfocal in character and remarkably mild in severity.

Lesions, even very large ones, dramatically decreased in size and in some cases completely resolved on T2-weighted fluidattenuated inversion recovery (T2-FLAIR) imaging. Interestingly, we found that lesions decreased both with and without administration of corticosteroids. Figure 2, I-K, shows an example of the near-complete resolution of large inflammatory lesions. To demonstrate the decrease in volume over time, 10 large lesions ( $n=1$ lesion per patient from 10 patients) were manually segmented, and the peak volume of the lesion was compared with the lesion volume on the patient's last available MRI. This analysis revealed that the lesions decreased in volume by an average of who had a history of at least 1 seizure included 1 patient with a static cortical heterotopia unrelated to CTLA4h status, 1 patient with posterior reversible encephalopathy syndrome (PRES), and 1 patient who had a biopsy-proven gliosarcoma.

Focal neurological deficits were rare and, if present, typically mild in severity. Thirteen of the 16 patients (81\%) with neuroinflammation had at least $1 \mathrm{MRI}$ with evidence of 1 or more new contrast-enhancing lesions without associated clinical symptoms. Interestingly, upon screening of CNS MRIs at the NIH, 2 patients were found to have neuroinflammatory lesions that included spinal cord involvement; neither patient exhibited clinical symptoms. The exceptions to this clinicoradiological dissociation were 2 patients who developed reduced bowel and/or bladder control with lower-extremity numbness and weakness suggesting a moderate-to-severe myelopathy. Both patients had expansile thoracic spinal cord inflammation on MRI, and both showed remarkable recovery with only minor residual deficits. The neurological symptoms experienced by this cohort are summarized in Table 1.

\section{CTLA4h-related neuroimaging findings}

Large, recurrent, multifocal, and inflammatory brain and spinal cord lesions. Fourteen patients (28\% of the cohort) had at least 1 MRI scan showing a contrast-enhancing intraparenchymal lesion in the CNS. In 10 of these patients, neuroinflammation was confirmed by biopsy (see Histopathological data in Methods for further details). Lesions were located in the supratentorium (14 of 14 cases, $100 \%$ ), infratentorium ( 9 of $14,64 \%)$, and spinal cord $(n=$ 8 of 12 cases with spinal cord imaging, 67\%). Lesions were multifocal in 13 of 14 patients (93\%) and were of various sizes. The volume of the largest supratentorial lesion for each patient ranged from $1.9 \mathrm{~mL}$ to $137.2 \mathrm{~mL}$. We observed very large lesions (>10 mL brain tissue) in 8 patients. Spinal cord lesions were extensive in
97\%, from $11.4 \mathrm{~mL}$ to $0.25 \mathrm{~mL}$ (Figure $2 \mathrm{~L}$ ).

Most patients (11 of 14, 79\%) developed new lesions on serial imaging, and many of the contrast-enhancing lesions showed persistent contrast enhancement over several months. Figure 3 depicts the episodes and persistence of CTLA4h-related neuroinflammation. The median proportion of MRI scans with 1 or more new lesions was $46 \%$ (range: $25 \%-88 \%$ ). The median proportion of MRI scans with 1 or more new or persistent contrast-enhancing lesions was $85 \%$ (range: $58 \%-100 \%$ ). This data set includes patients who underwent numerous MRIs and serial scans over time (up to 40 MRIs spanning 10 years of follow-up for some patients). These radiological findings occurred in the presence of variable treatments including acute and chronic corticosteroids and immune-modulatory therapies.

Leptomeningeal inflammation. Among the 14 patients with intraparenchymal lesions, 9 (64\%) had leptomeningeal contrast enhancements (LMEs) that changed in location, number, and/ or extent on serial imaging. One patient had stable LME on serial imaging. Interestingly, LME clearly preceded the formation of parenchymal lesions adjacent to the site of LME in some patients (Figure 4). In the 2 patients' images shown, we observed parenchymal inflammation 11 days (Figure 4, A-D) and 120 days (Figure 4, E, F, K, and L) after the initial detection of LME. In the 36 patients without MRI evidence of parenchymal CNS inflammation, $8(22 \%)$ also had at least 1 focus of LME. Unlike in patients with CNS inflammatory lesions, these foci were stable in size and location by serial imaging and never associated with a parenchymal lesion (Supplemental Figure 1, A and B, yellow chevron. In this case series, stable LME was not considered to definitively indicate the presence of neuroinflammation.

Other CNS imaging findings. In the 34 patients without MRI or CSF evidence of CNS inflammation, we found that 10 (29\%) 

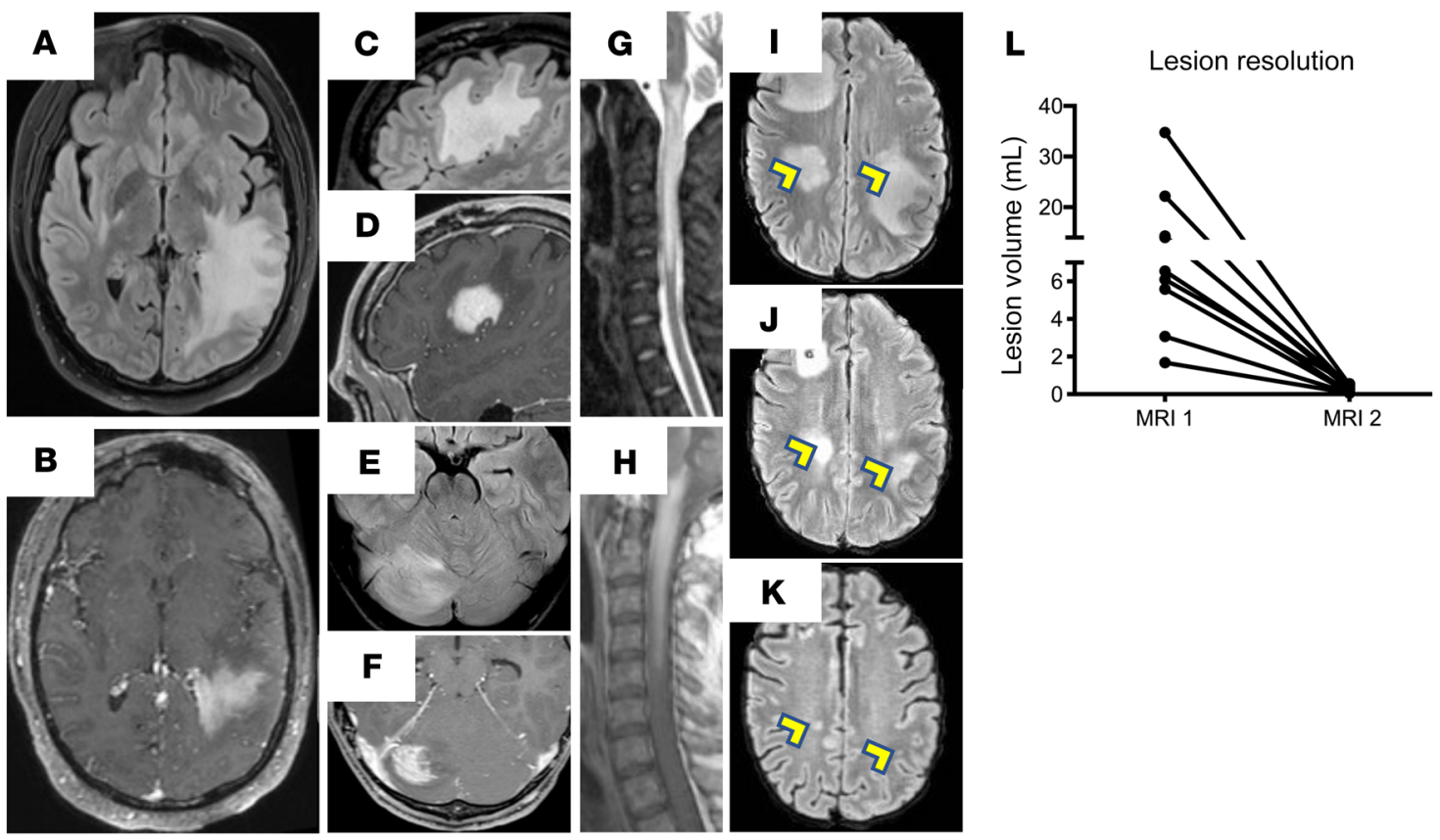

Figure 2. Representative CTLA4h MRI findings in patients with parenchymal inflammatory lesions. Patient numbers given here correspond to the data in Table 2. (A and B) Patient 2 showed worsening migrainous headaches with papilledema. T2-FLAIR image (A); T1-weighted contrast-enhanced image (B). (C and D) Patient 7 showed a focal motor seizure with secondary generalization and trace left triceps weakness. T2-FLAIR image (C); T1-weighted contrast-enhanced image (D). (E and F) Patient 5 had new headaches and a normal neurological examination. T2-FLAIR image (E); T1-weighted contrastenhanced image (F). ( $\mathbf{G}$ and $\mathbf{H}$ ) Patient 2 had a return of migrainous headaches following cessation of chronic immune-suppressive medication and a normal examination. Images in $\mathbf{G}$ are T2-weighted spinal cord images, and images in $\mathbf{H}$ are T1-weighted contrast-enhanced spinal cord images. (I-K) Serial MRI scans of patient 4. Time from scan in I to scan in J: 1 month; time from scan in J to scan in K: 1 year. (L) Change in lesion volume from maximum volume (MRI 1) to last available MRI (MRI 2).

patients, who were considered free of CNS disease, had small foci of abnormal signal in subcortical and deep white matter, which are generally not considered to be specific for a particular pathophysiological process. (Supplemental Figure 1, A-D, blue chevrons). These lesions did not enhance with contrast, nor did they change in size. We observed no new lesions over a median serial follow-up of 1.6 years (range: $0.2-4.8$ years).

Three patients had other significant imaging findings, including the following: (a) 1 patient had a retroorbital mass causing compression of the optic nerve and proptosis (Supplemental Figure 1, E and F); (b) 1 patient had a contrast-enhancing mass in the left temporal lobe, which, on biopsy and subsequent resection, was diagnosed as a gliosarcoma (Supplemental Figure 1, G and H); and (c) 1 patient had a bilateral occipital white matter signal abnormality characteristic of PRES (Supplemental Figure 1, I and J).

CSF

Fourteen patients (28\%) underwent CSF analysis, including 12 patients with brain and/or spinal cord lesions on MRI that were thought to be inflammatory, and 2 patients without contrastenhancing lesions on MRI, who underwent lumbar puncture (LP) to determine the cause of the headaches.

CSF WBC count and protein concentrations, as well as the presence or absence of CSF-specific OCBs, are provided in Table 2. Immunomodulatory treatment, including acute or chronic corticosteroid therapy, at the time of CSF analysis, and the timing of the LP in relation to the MRI findings, were variable.
Of the 12 patients with CNS inflammatory lesions on MRI, 11 had CSF leukocytosis with a mean WBC count of 50 cells/ $\mu \mathrm{L}$ (range: 1-185 cells/ $\mu \mathrm{L}$; normal range: $0-4$ cells $/ \mu \mathrm{L}$ ) and a differential of predominantly lymphocytes (mean, $88 \%$; range: $58 \%-99 \%$ ). The number of WBC did not correlate with the presence or absence of lesions on MRI. For example, the patient with the highest CSF pleocytosis $(185 \mathrm{WBC} / \mu \mathrm{L})$, although symptomatic with severe headaches and nausea, had a normal MRI at the time of LP. At follow-up 6 months later, a large inflammatory CNS lesion was observed on MRI, and clinically the patient complained of mild headaches. By contrast, the patient with the lowest CSF WBC count $(1 \mathrm{WBC} / \mu \mathrm{L})$ had numerous contrast-enhancing lesions on MRI. The mean CSF protein concentration was $70 \mathrm{mg} / \mathrm{dL}$ (range: $25-152 \mathrm{mg} / \mathrm{dL}$; normal: $15-45 \mathrm{mg} / \mathrm{dL}$ ). An elevated CSF protein concentration typically occurred in conjunction with elevated CSF pleocytosis.

We assessed OCB status in 10 patients and found that $6(60 \%)$ patients had OCBs in the CSF that were not present in serum. These OCBs either resolved or changed patterns in 4 patients who required serial LPs. Cytopathologic analysis of CSF, performed for 8 patients, was negative for malignancy. Microbiologic PCR analysis of CSF was negative for viruses (EBV, varicella zoster virus, human herpes viruses 6 and 7, herpes simplex virus, CMV, JC virus, adenovirus, and enterovirus). We detected no bacterial, fungal, or mycobacterial pathogens by special stains and cultures.

Flow cytometry was performed on CSF and peripheral blood from 10 patients who had neuroinflammatory lesions on MRI and from 2 additional patients who did not, and the results were com- 


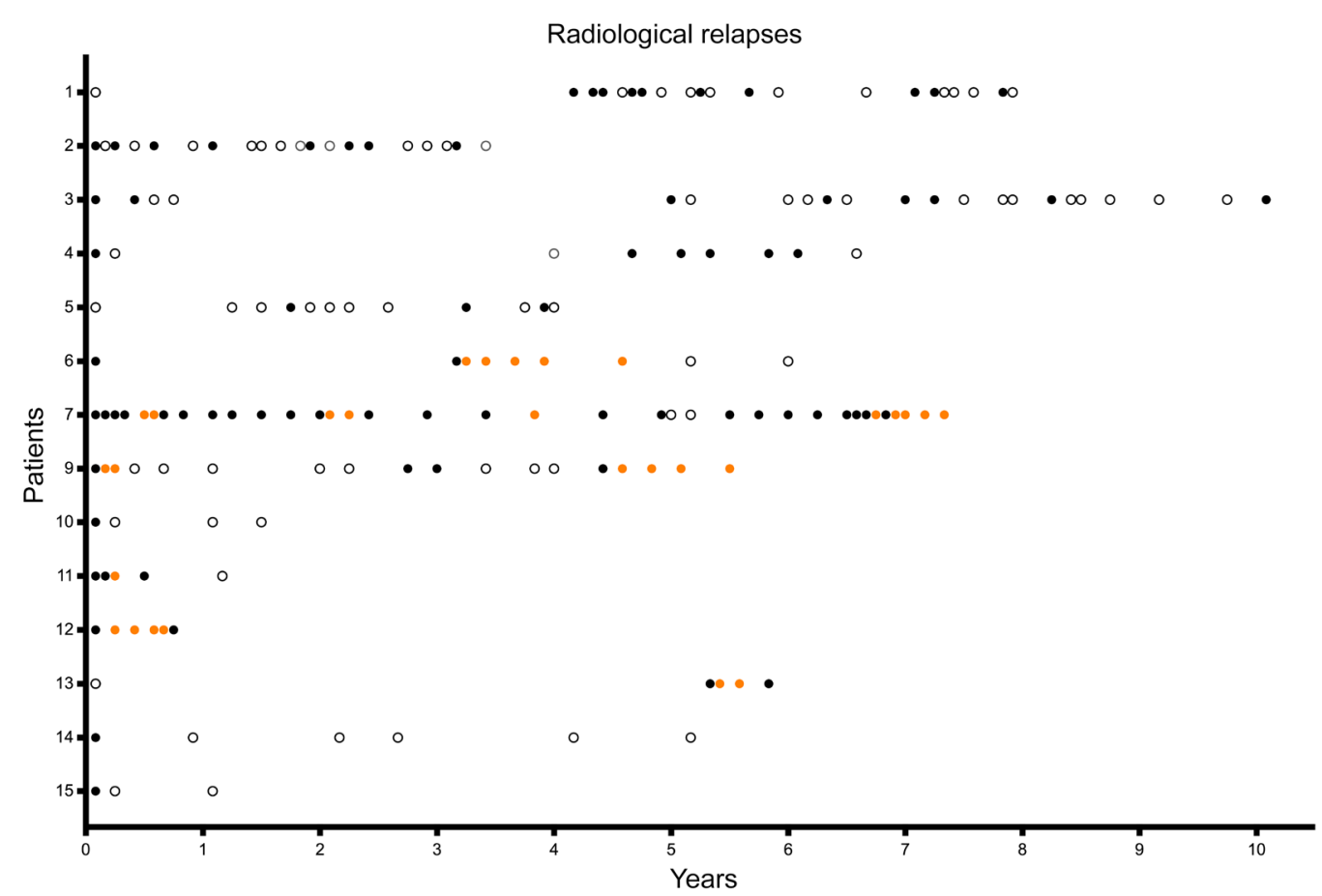

Figure 3. CTLA4h neuroinflammation can relapse at high frequency. Patient numbers given here correspond to the data in Table 2. Serial MRI scans were compared for new and contrast-enhancing lesions. Black circles indicate MRI scans with new T2-FLAIR or contrast-enhancing lesions; white circles indicates MRI scans without new T2-FLAIR or contrast-enhancing lesions; and orange circles indicate MRI scans without a new lesion but with persistent contrast enhancement.

pared with a pool of 31 healthy donors (normal donors [NDs]). The demographics for both groups and cell concentrations are shown in Supplemental Table 2. LP was performed within 2 weeks of detection of a contrast-enhancing lesion in all but 1 patient, who had MRI evidence of neuroinflammation 2 years prior. We quantified lymphocyte populations from blood and CSF using flow cytometry. The frequency of $\mathrm{CD} 4^{+} \mathrm{T}$ cells in blood or CSF did not differ between cohorts, but we detected fewer CD $8^{+} \mathrm{T}$ cells in the CSF $(P=0.0155)$ and a higher $\mathrm{CD} 4^{+} / \mathrm{CD}^{+} \mathrm{T}$ cell ratio in the $\mathrm{CSF}$ of patients in the CTLA4h cohort $(P=0.0101$; Figure $5 \mathrm{~A})$. The frequency of memory $\left(\mathrm{CD} 45 \mathrm{RA}^{-} \mathrm{CD} 27^{+}\right) \mathrm{CD}^{+} \mathrm{T}$ cells was elevated in the peripheral blood only in the CTLA4h cohort $(P=0.0009)$. Interestingly, the percentage of $\mathrm{CD} 4^{+} \mathrm{T}$ cells that are classified as memory follicular helper T (Tfh) cells (CD45RA-CXCR5 ${ }^{+}$) was elevated in the CTLA4h cohort, in both CSF and peripheral blood ( $P<0.0001$; Figure $5 B)$. The number of $\mathrm{B}$ cells as a proportion of the total lymphocyte population was lower in blood of patients in the CTLA4h cohort $(P=$ 0.0032) but not the CSF. B cell populations were highly variable in the CTLA4h cohort, in both peripheral blood and CSF, which may partially be due to prior treatment using B cell-depleting agents. However, on average, the proportion of CSF switched memory B cells (CD27+IgD-) was low in both blood and CSF (Figure 5C). Additional flow cytometric immune cell subsets and representative flow data are provided in Supplemental Figure 2.

Of the 2 patients with no prior contrast-enhancing MRI lesions who underwent LP, 1 had pleocytosis of $7 \mathrm{WBC} / \mu \mathrm{L}$ with normal protein and OCBs that were partially matched to the serum. The other patient had $2 \mathrm{WBC} / \mu \mathrm{L}$ and normal protein, but also had OCBs unique to CSF, suggesting compartmentalized CNS inflam- mation. The flow cytometric data for these 2 patients are included in Figure 5 and shown as orange dots.

\section{Neuropathology}

Ten of the 14 patients (71\%) with neuroinflammatory brain lesions had brain biopsies, and 1 patient underwent 2 biopsies separated by 2 years. All biopsies were obtained from anatomical regions that showed contrast enhancement on MRI and were performed at a variety of hospitals outside of the NIHCC. Seven biopsies were from cortex, which included both gray and white matter regions; 3 biopsies were from deep white matter; and 1 biopsy was from the cerebellum. The number and quality of the histological slides and the immunohistochemical stains performed on the biopsy specimens were highly variable.

All biopsy samples showed a mixed inflammatory infiltrate, predominately comprised of lymphocytes, histiocytes/macrophages, and plasma cells (Figure 6, A-P). Eosinophils, polymorphonuclear neutrophils, and dendritic cells were rarely seen. The extent of infiltration and ratio of cells and cell types varied between samples, even within different regions of the same biopsy. All samples showed perivascular infiltrates with prominent cuffing and variable degrees of patchy parenchymal infiltration (Figure 6, $A-G)$. Intraparenchymal inflammatory infiltrates predominantly involved the white matter, but also affected the gray matter and, when included in the biopsy, the meninges. The inflammatory infiltrates were rich in $\mathrm{CD}^{+} \mathrm{T}$ cells, of which $\mathrm{CD} 4^{+} \mathrm{T}$ cells dominated. We found that the extent of $\mathrm{CD} 2 \mathrm{O}^{+} \mathrm{B}$ cell infiltration was variable among patients, yet less prominent than that of the $\mathrm{T}$ cells (Figure 6, C and D). Of note, 4 patients' biopsies were obtained 
Table 2. Features of CTLA4h-associated neuroinflammation

\begin{tabular}{|c|c|c|c|c|c|c|c|}
\hline \multirow[b]{2}{*}{$\begin{array}{l}\text { Patient } \\
\text { no. }\end{array}$} & \multicolumn{7}{|c|}{ Neuroinflammation } \\
\hline & $\begin{array}{c}\text { MRI: } \\
\text { intraparenchymal }\end{array}$ & $\begin{array}{l}\text { MRI: } \\
\text { LME }\end{array}$ & $\begin{array}{l}\text { MRI: } \\
\text { recurrent }\end{array}$ & Pathology & $\begin{array}{c}\text { CSF: maximum WBC/ } \mu \mathrm{L} \text { CSF } \\
\text { (range) }\end{array}$ & CSF: OCB & $\begin{array}{l}\text { CSF: maximum protein/mg/dL } \\
\text { (range) }\end{array}$ \\
\hline 1 & + & + & + & + & $40(3-70)$ & + & $65(47-121)$ \\
\hline 2 & + & + & + & + & $29(4-148)$ & + & $122(97-152)$ \\
\hline 3 & + & + & + & + & $17(13-24)$ & + & $58(47-68)$ \\
\hline 4 & + & + & + & + & 13 & + & 66 \\
\hline 5 & + & + & + & A & $41(6-185)$ & + & $35(27-75)$ \\
\hline 6 & + & - & + & + & $21(15-28)$ & A & $32(27-35)$ \\
\hline 7 & + & + & + & + & $4(1-61)$ & + & $44(31-72)$ \\
\hline 8 & - & - & - & A & 2 & + & 22 \\
\hline 9 & + & + & + & + & $32(27-36)$ & A & $19(18-20)$ \\
\hline 10 & + & + & + & + & 5 & - & 27 \\
\hline 11 & + & + & + & $+^{B}$ & 16 & - & 77 \\
\hline 12 & + & - & + & A & 1 & - & 27 \\
\hline 13 & + & + & + & A & A & A & A \\
\hline 14 & + & - & - & + & A & A & A \\
\hline 15 & + & - & + & + & 7 & - & 35 \\
\hline 16 & - & - & - & A & 7 & + & 50 \\
\hline
\end{tabular}

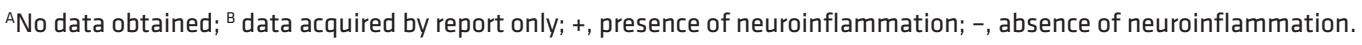

after anti-CD20 therapy. Most of the biopsies had a large plasma cell population clustered around the perivascular space and in the brain parenchyma (Figure $6 \mathrm{H}$ ). Immunoglobulin (Ig) staining showed an excess of either $\lambda$ chain or $\kappa$ light chain in 2 patients each. Biopsies from 2 patients showed a striking population of histiocytes with abundant crystalline and globoid structures, consistent with crystal-storing histiocytosis with IgM greater than IgG (Figure 6, I and J). We noted that histiocytes/macrophages were also present, but they lacked an activated or "foamy" morphology. In biopsies from 4 patients who had a primary lymphohistiocytic infiltrate, immunohistochemical stainings of glial fibrillary acidic protein (GFAP), a marker of astrocytic gliosis, and neurofilament, a marker of neuropil integrity, were relatively normal (Figure $6, \mathrm{~K}-\mathrm{M})$. In contrast, in 2 biopsy samples that had an extensive plasmacytic infiltrate, we observed increased GFAP staining and reduced neurofilament staining, indicating that some tissue injury can occur (Figure 6, N-P).

\section{Treatment}

Patients with CTLA4h and neuroinflammatory brain lesions were treated with a variety of immunomodulatory agents, many chosen before their genetic diagnosis. Nearly all patients with neuroinflammatory lesions on MRI (12 of 14 cases, $86 \%$ ) had received immune-suppressive therapy before recognition of their CNS lesions. Common treatments included intermittent corticosteroids, mycophenolate mofetil, sirolimus, rituximab, and abatacept. Treatments for CNS inflammation and/or neurological symptoms commonly consisted of pulse doses (up to $1000 \mathrm{mg}$ ) of methylprednisolone. Acutely symptomatic patients, such as those with migrainous headaches, had rapid and sustained improvement in headache following intravenous steroid administration. We found no clear association between the frequency or severity of neurologic symptoms, such as headaches, and the type of chronic immune-modulatory therapy. The lack of standardized treatments in this natural history cohort limits the ability to identify any pattern of treatment effect.

Of particular interest is a case involving a 30-year-old man (patient 2 in Table 2) who had large neuroinflammatory lesions on MRI (Figure 2, A and B) and was followed at the NIH for 3 years. He was treated with sirolimus and abatacept, but shortly after these therapies were stopped because of a local skin infection, he developed a significantly large cervical spinal cord inflammatory lesion (Figure 2, G and $\mathrm{H}$ ). The only symptom he reported was headache, and his neurological examination was normal. He was placed on high-dose intravenous corticosteroids, and his headache completely resolved by the next morning. His longitudinally extensive cervical spine lesion resolved over 3 months with little residual signal abnormality.

\section{Discussion}

CTLA4h is a rare, genetic cause of systemic immune dysregulation with the potential to cause severe recurrent neuroinflammation. Among our cohort of 50 patients carrying uniallelic mutations, 14 patients had neuroinflammatory lesions seen on MRI, and 2 additional patients had mild inflammation only observed on CSF analysis. All the patients with CNS involvement also had evidence of immune-mediated disease in other organs or autoimmunity; i.e., none had isolated CNS disease.

Our findings systematically characterizing CNS involvement in patients with CTLA4h revealed several findings. First, the neuroinflammatory lesions were often dramatic in size (Figure 2, A-H, dynamic over time (Figure 2, I-L), and recurrent (Figure 3), and they progressed from the leptomeningeal space into the brain parenchyma (Figure 4). Second, inflammatory changes in the CSF sometimes preceded the parenchymal infiltration observed on MRI and sometimes occurred without intraparenchymal disease. 

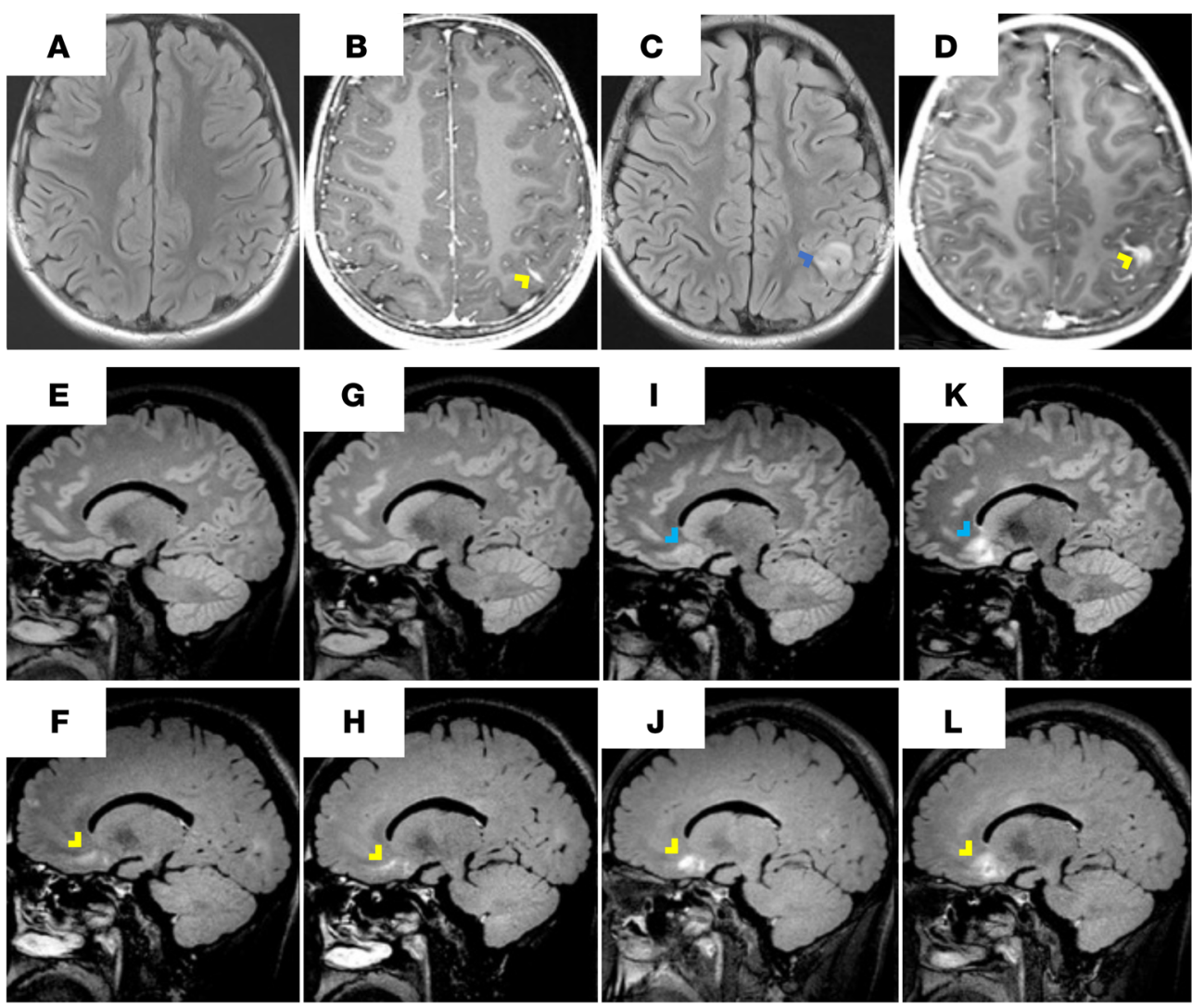

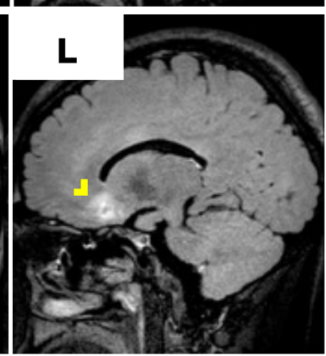

Figure 4. CTLA4h-associated neuroinflammation often starts in the leptomeninges. LME on MRI can precede parenchymal lesion formation. Patient numbers given here correspond to the data in Table 2. (A-D) Patient 9 developed headaches and a complex partial seizure attributed to a mesial temporal lobe lesion (not shown). MRI scans at clinical onset (A and $\mathbf{B}$ ) and 11 days later (C and $\mathbf{D}$ ) show a fast temporal evolution of LME (yellow chevron) to a parenchymal lesion (blue chevron). T2-FLAIR images ( $\mathbf{A}$ and $\mathbf{C}$ ); T1-weighted images after contrast (B and D). (E-L) Patient 13 was clinically asymptomatic and had extensive LME (yellow chevrons) on sequential MRIs, each done approximately 1 month apart. T2-FLAIR images (E, G, I, and K); T2-FLAIR images after contrast (F, $\mathrm{H}, \mathrm{J}$, and $\mathrm{L}$ ).
Interestingly, most $\mathrm{T}$ and $\mathrm{B}$ cell subsets, including markers of activation in the blood and CSF, were similar between patients with CTLA4h and NDs. Third, the radiological findings were consistent with the pathology of a primarily nondestructive inflammatory infiltrate of the brain and meninges (Figure 6).

A key observation was that clinical findings (headaches and seizure), although discordant with the extent and location of the inflammatory lesions, also supported this nondestructive pathophysiology. A notable exception were large lesions in the thoracic spinal cord observed in 2 patients, which caused significant neurological symptoms. However, the symptoms were more consistent with compressive myelopathy and not direct tissue injury within the cord, and both of these patients made remarkable recoveries. The extent of this striking clinicoradiologic dissociation is extreme among immune-mediated neurological diseases. Indeed, even in other conditions, such as multiple sclerosis and neurosarcoidosis, in which clinical signs and symptoms do not necessarily accompany radiological findings, the size and degree of active inflammation seen on MRI scans in patients with CTLA4h with essentially normal neurological exams is unusual.

Variable penetrance in CTLA4h has been reported in small case series $(16,17)$ and was observed in this cohort. The most extreme example in this cohort was a pair of identical twins, one of whom had multiorgan immune-mediated disease, including CNS inflammation, while the other twin was asymptomatic. There are no known modifier genes or environmental influences that determine in whom or in which organs CTLA4h-associated inflammation will develop. None of the clinically asymptomatic patients in this cohort developed new inflammatory disease during the period of observation of this study. In patients with demonstrable CNS inflammation, the factors that drive disease persistence and relapses are also unknown. All patients with neuroinflammatory lesions observed on MRI had evidence of CTLA4h-related inflammation affecting multiple organ systems. Although there was great variability in the specific pattern, the digestive, pulmonary, and hematopoietic systems were most commonly affected. However, there were many patients without overt CNS inflammation who had CTLA4h-associated inflammation affecting these same organ systems. Interestingly, when we observed new CNS inflammation, we found no worsening of symptoms in other organ systems either immediately before, during, or shortly after a CNS relapse. These observations suggest compartmentalization of organ-specific inflammatory responses in CTLA4h.

One caveat is that the nonspecific clinical symptoms in this patient population and the dramatic spontaneous resolution of lesions seen on MRI follow-up, or with systemic corticosteroid treatment, may have a propensity to mask the prevalence of asymptomatic CNS disease and lead to a delay in its recognition. Since we observed both mild CSF pleocytosis or OCBs on CSF analysis without radiological evidence of CNS inflammation (patients 8 and 16) and CSF inflammation preceding the development of a MRI-visible lesion (patient 5), we suspect that inflammation limited to CSF may exist as a form of neurological disease and might proceed to parenchymal or meningeal involvement in only a subset of patients. Within this spectrum, patients with radiological evidence of CNS lesions may represent the more severe manifestation of disease. Nevertheless, infiltrative CNS inflammation does not exist as an isolated entity in an otherwise asymptomatic 
A $T$ cell frequency and ratio CD4 ${ }^{+} \mathrm{T}$ cell
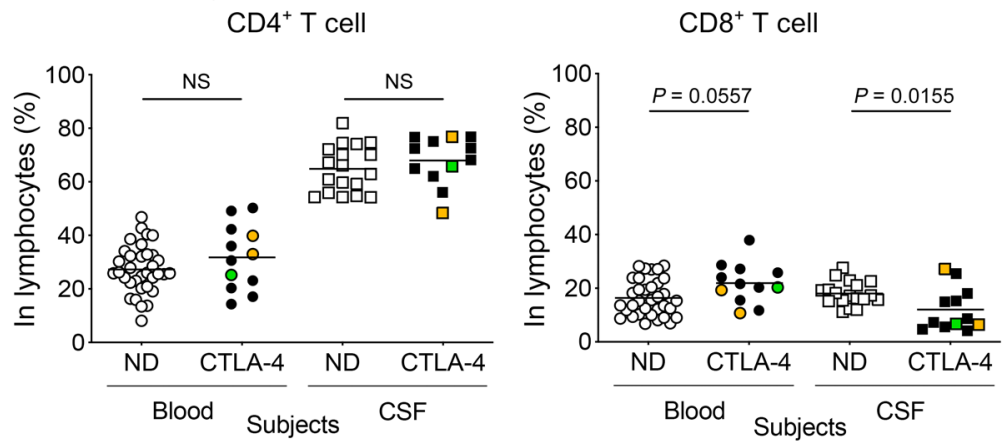

B T cell subsets

CD4 ${ }^{+}$Memory $\mathrm{T}$ cell
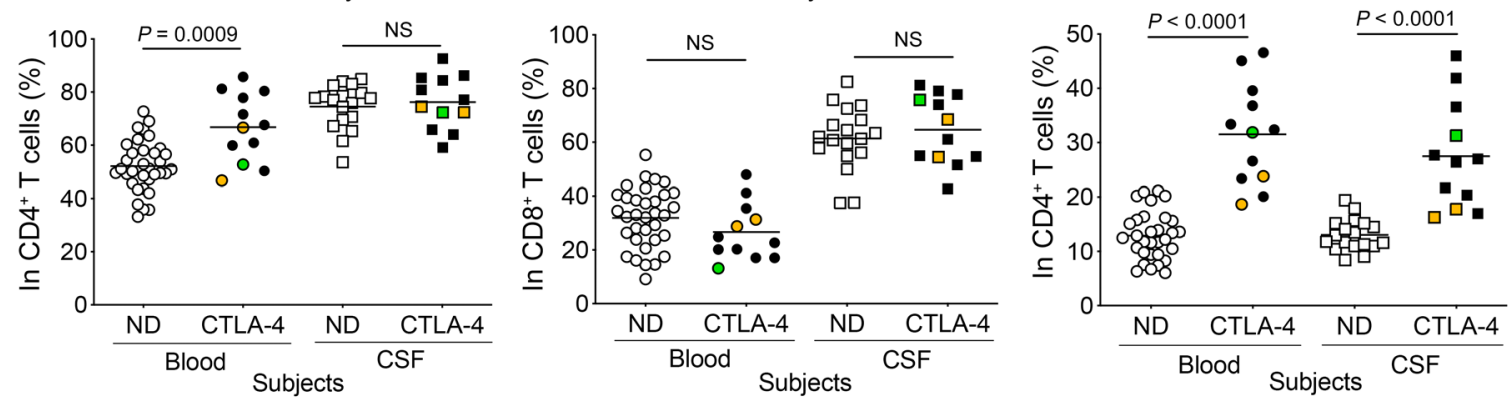

$B$ cell frequency and subsets
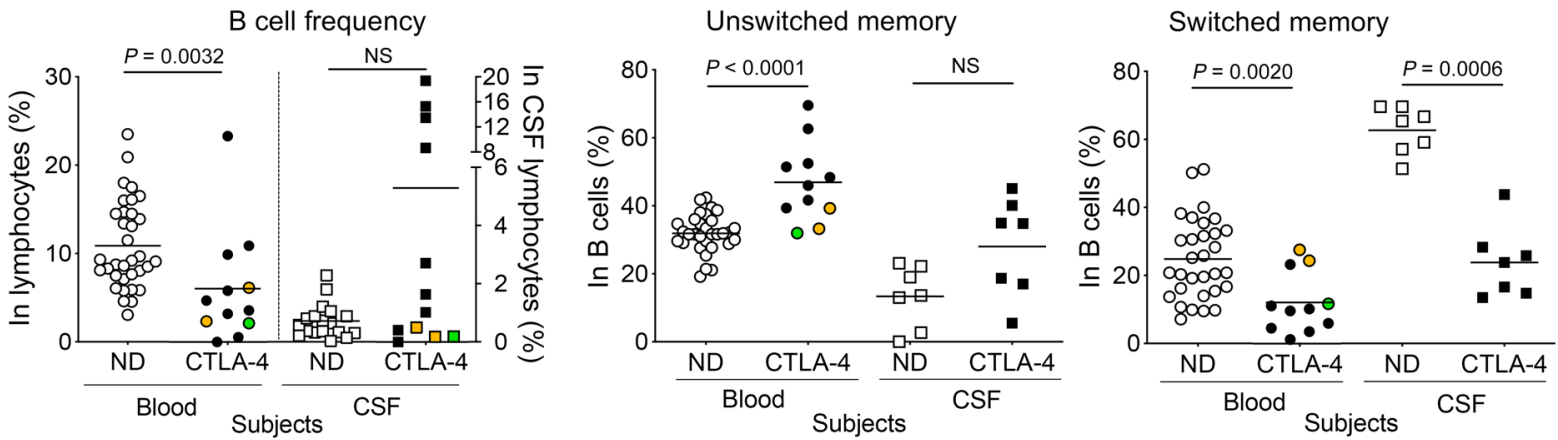

Figure 5. CSF is inflamed in CTLA4h. Flow cytometric data comparing patients with CTLA4h with active neuroinflammatory lesions on MRI (black), a prior history of neuroinflammatory lesions (green), and no history of inflammatory lesions (orange) on MRI with a cohort of healthy controls (NDs, white circles). All graphs depict values from blood (left half of each panel) and CSF (right half). (A) Total frequency of CD4 ${ }^{+}$and CD8 ${ }^{+}$T cells within total lymphocyte counts and the ratio of CD4 $4^{+}$to $C D 8^{+} T$ cells. (B) Percentage of memory $T$ cell subtypes (CD45RA- $\left.C D 27^{+}\right)$in CD4 $4^{+} T$ cells and $C D 8^{+} T$ cells, and memory Tfh cells (CD45RA-CXCR5') in CD4+ $T$ cells (right). (C) Frequency of total B cells and memory B cell subtypes, including unswitched memory (IgD+CD27+) and switched memory (IgD-CD27+) B cells. For all flow cytometric data, the Mann-Whitney $U$ test was performed for comparisons between ND and CTLA4 cohorts, within each compartment (i.e., peripheral blood and CSF). Significance was set at $P<0.05$. Each flow cytometric experiment for lymphocyte subsets from peripheral blood that was paired with a CSF sample obtained at the same time represents a single experiment, given the nature of the specimen (CSF obtained by LP) and the limited amount of specimen that could be obtained, as well as the sensitivity for the timing of the procedure according to the clinical status and the treatment course of the patient.

mutation carrier. Disease-defining biomarkers and specific autoantibodies in CSF remain to be explored and await correlation with clinical phenotypes and radiological markers.

Genetic CTLA4h disease in humans has important differences compared with the published literature of neurological side effects from anti-CTLA4 therapy used for the treatment of various malignancies and compared with published animal studies of Ctla4-KO mice. CNS infiltrates have not been reported in published studies of Ctla4-KO mice. This may stem from the fact that Ctla4-KO mice typically survive for only approximately 3 weeks and die as a result of massive lymphocytic infiltrates in the heart, lungs, and pancreas $(15,18)$, thus not allowing enough time for CNS disease to develop. Interestingly, Ctla4-heterozygous mice lack any clinical phenotype $(18,19)$, whereas in humans, there is variable penetrance of clinical symptoms, even within the same family. Autoimmune conditions, including myasthenia gravis, inflammatory myopathies, and acute demyelinating polyneuropathy, have been described in some patients receiving checkpoint inhibitor therapies, including anti-CTLA4 antibodies, whereas a subset of patients do not develop neuroimmunological manifestations, either centrally or periph- 

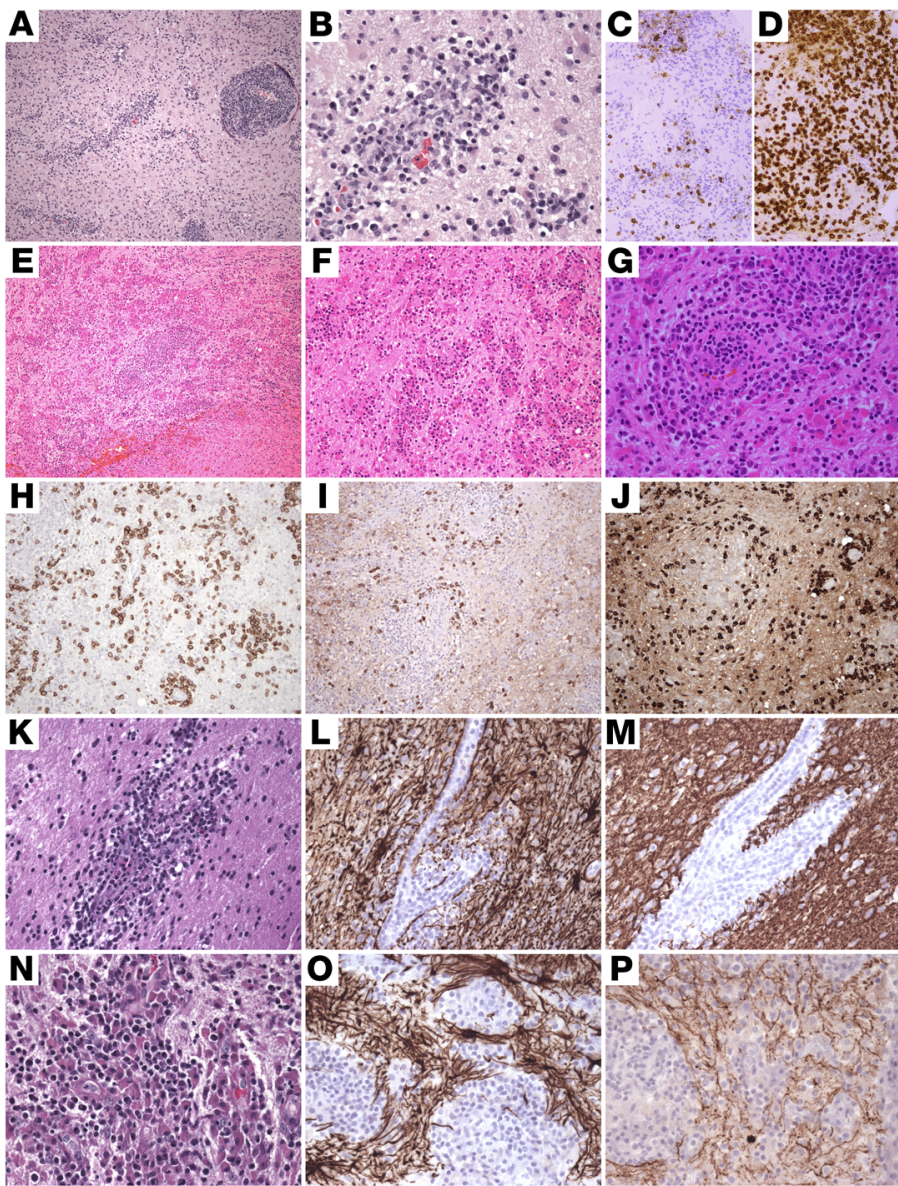

erally $(5,7,8)$. Our cohort did not show evidence of autoimmune disease affecting the PNS. Previously reported anti-CTLA4-related CNS disease includes sarcoid-like inflammation, necrotizing myelopathy, PRES, hypophysitis, and aseptic meningitis $(5,6,8$, 9, $14,20)$. Of these entities, PRES was seen in 1 of our patients, but this patient did not meet our criteria for neuroinflammatory lesions because of a lack of contrast enhancement. In the published case reports, severe immune-mediated CNS disease was described, but with much more destructive injury than that seen in our cohort. Although the mechanism behind this difference is unclear, it may be related to preexisting differences in overall immune function.

There are limitations in the interpretation of the findings from this study. This was a natural history study, and much of the data were retrospectively collected from charts compiled before diagnosis of CTLA4h. Increased awareness of the clinical patterns observed in patients with CTLA4h will allow for quicker diagnosis and the ability to conduct a more prospective analysis of clinical and paraclinical information. Future natural history studies of patients with CTLA4h and other genetic immune dysregulation syndromes may elucidate the mechanism of CNS immune privilege, as well as communication between the immune system and the immune CNS-resident effectors of immunity in health and disease.

\section{Methods}

Patient population. The patients reported here were selected from a cohort of 96 patients from 32 families with a genetically defined
Figure 6. Brain biopsy shows inflammation in CTLA4h brain lesions. (A-D) Right frontal deep white matter lesion showing cellular infiltrate on $\mathrm{H} \& \mathrm{E}$ staining, with infiltration of $\mathrm{CD} 2 \mathrm{O}^{+} \mathrm{B}$ cells $(\mathbf{C})$ and $\mathrm{CD} 3^{+}$ T cells (D). Original magnification, $\times 100$ (A) and $\times 400$ (B-D). (E-J) Left parietal leukocortical white matter lesion showing cellular infiltrate on H\&E staining, with evidence of CD138 plasma cells $(\mathbf{H})$ and both $\operatorname{IgC}(\mathbf{I})$ and IgM (J) production. Original magnification, $\times 100(\mathbf{E}), \times 200(\mathbf{F}$ and $\mathbf{H}-\mathbf{J})$, and $\times 400(\mathbf{G})$. (K-M) Right frontal white matter lesion and (N-P) right occipital white matter lesion showing cellular infiltrate on H\&E staining ( $\mathbf{K}$ and $\mathbf{N}$ ), with variable degrees of astrocytosis ( $\mathbf{L}$ and $\mathbf{0})$ and preserved neuropil (M and $\mathbf{P}$ ). Original magnification, $\times 200$.

mutation in the CTLA4 gene leading to reduced expression of the CTLA4 protein. The methods for genetic testing and evaluation of CTLA4 expression have been described previously (16). Fifty patients from 23 families, for whom both MRI scans and clinical data were available to us, were included in this analysis.

Clinical data. Medical records of all 50 patients were reviewed, with a focus on neurological clinical histories. Demographics, including sex, current age, and age at the time of the first presumed CTLA4h-mediated clinical manifestation, were recorded. For each patient, we also recorded the major systemic clinical manifestations of CTLA4h-mediated disease that have previously been reported, including autoimmune cytopenia, hypogammaglobinemia, and infiltrative lymphocytosis of major organs, and other autoimmunity-mediated clinical diseases (e.g., diabetes mellitus type 1, thyroiditis, psoriasis). Neurological symptoms and clinical examination details were collected at each visit or noted from historical clinical records.

Radiological data. All available MRI scans, including studies from outside institutions, were reviewed by a single boardcertified neurologist with expertise in neuroimaging. All 50 patients underwent at least 1 brain MRI scan (total brain MRI scans: 289), and 20 patients also had at least 1 spinal cord MRI scan (total spinal cord MRI scans: 54 ). The median number of brain MRI scans reviewed per patient was 3 (range: 1-40), and the median number of spinal cord MRI scans was 1 (range: 1-16). Serial brain MRI scans were available for review for 34 patients over a median of 2.3 years (range: 0.2-10.1 years). Serial MRI scans of the spinal cord were available for review for 10 patients over a median of 1.6 years (range: $0.4-4.3$ years).

Given the rarity of the disease and the wide geographic distribution of the patients, MRI scans were performed on machines from various MRI manufacturers and were acquired using various field strengths and sequence acquisition parameters. In general, sequences including T2-weighted, T2-weighted fluid-attenuated inversion recovery (FLAIR), and post-gadolinium T1-weighted (T1c) were available; these scans, as well as post-gadolinium T2-FLAIR (Fc), when available, were visually inspected for evidence of lesions. Inflammation was strictly defined by the presence of contrast-enhancing parenchymal lesions in the CNS. On all scans, the location of lesion(s) (supratentorium, infratentorium including the cerebellum, and spinal cord) was noted. For patients who underwent serial MRI studies, the appearance of new lesions was noted. T1c and, when available, Fc sequences were assessed for abnormal parenchymal and leptomeningeal contrast enhancement (LME) (21).

In patients with imaging evidence of contrast-enhancing parenchymal brain lesions, T2-FLAIR, T1c, Fc, and any other available 
sequences were run through an image-processing pipeline that included image registration (Advanced Normalization Tools [ANTs], version 2.1) (22) across serial scans for each patient, followed by registration of the aligned scans to a common atlas space (Montreal Neurological Institute Atlas). This process facilitated comparison of scans over time to identify new lesions. For each patient, the largest lesion observed on the serial T2-FLAIR scans was manually segmented using Medical Image Processing, Analysis, and Visualization (MIPAV) software, version 7.02 (http://mipav.cit.nih.gov) to generate volumetric data; this allowed identification of the peak volume and amount of lesion resolution on the last available MRI.

Histopathological data. All available brain biopsy samples $(n=$ 10) were evaluated. Patients with brain biopsies are noted in Table 2. The presence and predominant cell types found in any cellular infiltrate were noted.

All biopsies were from formalin-fixed, paraffin embedded tissues and stained with H\&E. Immunohistochemical staining was performed using an Ultra automated immunostainer (Roche) according to the manufacturer's instructions. Antigen retrieval for each antibody listed below was performed according to the manufacturers' instructions with minor modifications. The following antibodies were used: CD20 (clone L26, predilute, 760-2531, Roche); CD3 (clone 2GV6, predilute, 790-4341 Roche); CD138 (clone B-838, predilute, 760-4248, Roche); CD4 (clone SP-35, predilute, 790-4423, Roche); CD8 (clone SP57, predilute, 790-4460); $\kappa$ (760-2514) and $\lambda$ (760-2515) light chains (rabbit polyclonal, Roche); IgM (rabbit polyclonal, A0425, Dako); IgG (rabbit polyclonal, A0423, Dako); neurofilament (clone 2F11, M0762, Dako); and GFAP (clone EP672Y, predilute, 760-4345). Antibodies were detected with the ultraVIEW Kit (Roche) using DAB as a chromogen.

Images were taken with an Olympus DP27 microscope with a U-TV0.63XC adapter and were imported with Olympus cellSens software and Adobe Photoshop CC 2019.

CSF data. Clinical CSF laboratory reports for 14 patients who had LPs performed at outside institutions or at the NIHCC were reviewed. Patients with CSF analyses are noted in Table 2. Differential cell counts, protein values, OCBs, cytology studies, and infection markers were variably available for review.

Immunophenotyping by flow cytometry was performed on peripheral blood and CSF from 12 patients with CTLA4h. Whole blood and CSF flow cytometric results in the CTLA4h cohort were compared with a historical cohort of NDs, who had samples collected for various research protocols. The demographics for the ND and CTLA4h cohorts are included in Supplemental Table 2. Whole-blood samples from 31 NDs and the matched CSF from 18 NDs were used for comparison with samples from the CTLA4h cohort.

Details on the flow cytometric methods used were previously described (23). For analysis of peripheral blood lymphocyte and CSF lymphocyte populations, EDTA-treated whole blood or CSF cells were stained with CD3 (clone UCHT1), CD4 (clone RPA-T4), CD8 (clone
SK1), CD14 (clone M5E2), CD16 (clone 3G8), CD19 (clone HIB19), CD25 (clone M-A251), CD27 (clone M-T271), CD45 (clone H130), CD45RA (clone HI100), CD56 (clone Bi59), CXCR5 (clone RF8B2), IgD (clone IA6-2), and HLA-DR (clone G46-6) (all from BD Biosciences) and PD-1 (clone EH12.2H7, BioLegend). Since B cells (CD45 ${ }^{+} \mathrm{CD}^{-}$ $\mathrm{CD}^{+}{ }^{+}$) were rarely detected in CSF, CSF samples from 7 NDs and 7 patients with CTLA4h were used for B cell subset analysis. Samples were acquired using an LSR II (BD Biosciences). The data were analyzed using FlowJo 10.2 software.

Statistics. Descriptive data, including demographic information, are presented as means and ranges. Statistical analysis of flow cytometric data was performed using GraphPad Prism (GraphPad Software). For comparisons between ND and CTLA4h with neuroinflammation groups, the Mann-Whitney $U$ test was used, and significance was set at $P<0.05$.

Study approval. All patients provided written informed consent for enrollment in this study. All research protocols were approved by the IRB of the NIHCC.

\section{Author contributions}

MKS conceptualized the study, curated data, performed formal analysis, and wrote the manuscript. SP conceptualized the study, curated data, and analyzed images. YEA curated the data and performed formal analysis. HCS, SJ, and VKR supervised the study and edited the manuscript. AR was responsible for project administration. IC supervised and conceptualized the study and edited the manuscript. DSR supervised the study, edited the manuscript, provided resources, and acquired funding. GU supervised and conceptualized the study, edited the manuscript, acquired funding, and performed genetic verification of disease.

\section{Acknowledgments}

We would like to thank the families and patients who took part in this study. We would also like to thank Steven Holland, who provided editorial feedback on the manuscript, and Pam Angelus for clinical support. This work was supported by intramural research funds from the NINDS and NIAID, NIH. MKS received Clinician Scientist Fellowship funding through the National Multiple Sclerosis Society-American Brain Foundation.

Address correspondence to: Daniel S. Reich, Translational Neuroradiology Section, National Institute of Neurological Disorders and Stroke (NINDS), NIH, Building 10, Room 5C103, 10 Center Drive, Bethesda, Maryland 20814, USA. Phone: 301.496.1801; Email: daniel.reich@nih.gov. Or to: Gulbu Uzel, Immunopathogenesis Section, Laboratory of Clinical Immunology and Microbiology, National Institute of Allergy and Infectious Disease (NIAID), NIH, Building 10, Room 12C103, 10 Center Drive, Bethesda, Maryland 20814, USA. Phone: 301.451.9035; Email: gulbu.uzel@nih.gov.
1. Linsley PS, Brady W, Urnes M, Grosmaire LS, Damle NK, Ledbetter JA. CTLA-4 is a second receptor for the $\mathrm{B}$ cell activation antigen B7.J Exp Med.1991;174(3):561-569.

2. Linsley PS, et al. Coexpression and functional cooperation of CTLA-4 and CD28 on activated T lymphocytes. JExp Med.1992;176(6):1595-1604.
3. Schiff M, et al. Efficacy and safety of abatacept or infliximab vs placebo in ATTEST: a phase III, multi-centre, randomised, double-blind, placebo-controlled study in patients with rheumatoid arthritis and an inadequate response to methotrexate. Ann Rheum Dis. 2008;67(8):1096-1103.
4. Hodi FS, et al. Improved survival with ipilimumab in patients with metastatic melanoma. $N$ Engl J Med. 2010;363(8):711-723.

5. Bertrand A, Kostine M, Barnetche T, Truchetet ME, Schaeverbeke T. Immune related adverse events associated with anti-CTLA-4 antibodies: systematic review and meta-analysis. BMC Med. 
2015;13:211.

6. Friedman CF, Proverbs-Singh TA, Postow MA. Treatment of the immune-related adverse effects of immune checkpoint inhibitors: a review. JAMA Oncol. 2016;2(10):1346-1353.

7. Wilgenhof S, Neyns B. Anti-CTLA-4 antibodyinduced Guillain-Barré syndrome in a melanoma patient. Ann Oncol. 2011;22(4):991-993.

8. Bot I, Blank CU, Boogerd W, Brandsma D. Neurological immune-related adverse events of ipilimumab. Pract Neurol. 2013;13(4):278-280.

9. Liao B, Shroff S, Kamiya-Matsuoka C, Tummala S. Atypical neurological complications of ipilimumab therapy in patients with metastatic melanoma. Neuro-oncology. 2014;16(4):589-593.

10. Thaipisuttikul I, Chapman P, Avila EK. Peripheral neuropathy associated with ipilimumab: a report of 2 cases. J Immunother. 2015;38(2):77-79.

11. Cao Y, et al. CNS demyelination and enhanced myelin-reactive responses after ipilimumab treatment. Neurology. 2016;86(16):1553-1556.

12. Williams TJ, et al. Association of autoimmune encephalitis with combined immune checkpoint inhibitor treatment for metastatic cancer. JAMA Neurol. 2016;73(8):928-933.

13. Gerdes LA, et al. CTLA4 as immunological checkpoint in the development of multiple sclerosis. Ann Neurol. 2016;80 (2):294-300.

14. Abdallah AO, et al. Ipilimumab-induced necrotic myelopathy in a patient with metastatic melanoma: A case report and review of literature. J Oncol Pharm Pract. 2016;22(3):537-542.

15. Tivol EA, Borriello F, Schweitzer AN, Lynch WP, Bluestone JA, Sharpe AH. Loss of CTLA-4 leads to massive lymphoproliferation and fatal multiorgan tissue destruction, revealing a critical negative regulatory role of CTLA-4. Immunity. 1995;3(5):541-547.

16. Kuehn HS, et al. Immune dysregulation in human subjects with heterozygous germline mutations in CTLA4. Science. 2014;345(6204):1623-1627.

17. Schubert D, et al. Autosomal dominant immune dysregulation syndrome in humans with CTLA4 mutations. Nat Med. 2014;20(12):1410-1416.
18. Waterhouse P, et al. Lymphoproliferative disorders with early lethality in mice deficient in Ctla-4. Science. 1995;270(5238):985-988.

19. Chambers CA, Sullivan TJ, Allison JP. Lymphoproliferation in CTLA-4-deficient mice is mediated by costimulation-dependent activation of CD4 ${ }^{+}$T cells. Immunity. 1997;7(6):885-895.

20. Fecher LA, Agarwala SS, Hodi FS, Weber JS. Ipilimumab and its toxicities: a multidisciplinary approach. Oncologist. 2013;18(6):733-743.

21. Absinta $\mathrm{M}$, et al. Leptomeningeal gadolinium enhancement across the spectrum of chronic neuroinflammatory diseases. Neurology. 2017;88(15):1439-1444.

22. Avants BB, Tustison NJ, Song G, Cook PA, Klein A, Gee JC. A reproducible evaluation of ANTs similarity metric performance in brain image registration. Neuroimage. 2011;54(3):2033-2044

23. Enose-Akahata $Y$, et al. Immunophenotypic characterization of CSF B cells in virusassociated neuroinflammatory diseases. PLOS Pathog. 2018;14(4):e1007042. 